\title{
Experimental Demonstration at 10Gbit/s of a 2R- Regenerator based on the Mutual Optical Feedback between a Laser Diode and an SOA
}

\author{
W. D’Oosterlinck, S. Verspurten, G. Morthier, R. Baets \\ Department of Information Technology, Ghent University - IMEC, Sint-Pietersnieuwstraat 41, B-9000 Gent, Belgium \\ Tel.: +32 9 2648930.,E-mail:wdooster@intec.ugent.be \\ M.K. Smit \\ COBRA Research Institute, Technische Universiteit Eindhoven, Postbus 513, 5600 MB Eindhoven, The Netherlands
}

\begin{abstract}
A new regenerator concept based on the feedback between a laser diode and an SOA has been tested using an integrated version. Excellent regenerator characteristics, both static and 10Gbit/s dynamic operation, have been obtained.

(C)2005 Optical Society of America

OCIS codes: (250.0250) Optoelectronics; (250.5980) Semiconductor Optical Amplifiers; (250.5300) Photonic Integrated Circuits
\end{abstract}

\section{Introduction}

All-optical regenerators could be a key building block of future all-optical networks. The key properties for a good all-optical regenerator are high-speed operation combined with a steep decision characteristic. Several regeneration schemes have already been proposed [1,2]. Most of them are based on the use of semiconductor optical amplifiers based interferometric set-ups. These schemes have the added advantage that they can easily be integrated on a single chip. It has however been shown that it is difficult to obtain both high-speed operation [3,4], bitrate independent operation and a steep decision characteristic [5,6] with the same component.

Earlier we presented a new type of regenerator $[7,8]$ that gives a steep decision characteristic without making use of interferometric structures. The regenerator is based on an SOA in a feedback scheme with a DFB-laser.

In this paper we present measurement results of the static and dynamic performance of this scheme. The measurements were performed on a component, meant as a wavelength-selectable laser diode [9], but especially suited also for our purpose. We show a very steep optical decision characteristic combined with high-speed operation. Since there is no interference involved in obtaining the optical decision characteristic the regenerator can be used over a wide wavelength region and is very stable against environmental changes.

\section{Principle of operation}

A schematic of the regenerator is shown in Fig. 1. The light coming from the SOA is partly coupled into the laser diode. The same fraction of the laser light is coupled back into the SOA. The part of the output power that is not coupled to the laser diode is coupled out. Simulations and measurements on a set-up consisting of a discrete SOA and laser diode showed a very steep static transfer function $[7,8]$. Since we use here an integrated version of the regenerator the roundtrip time needed for the feedback is reduced significantly.

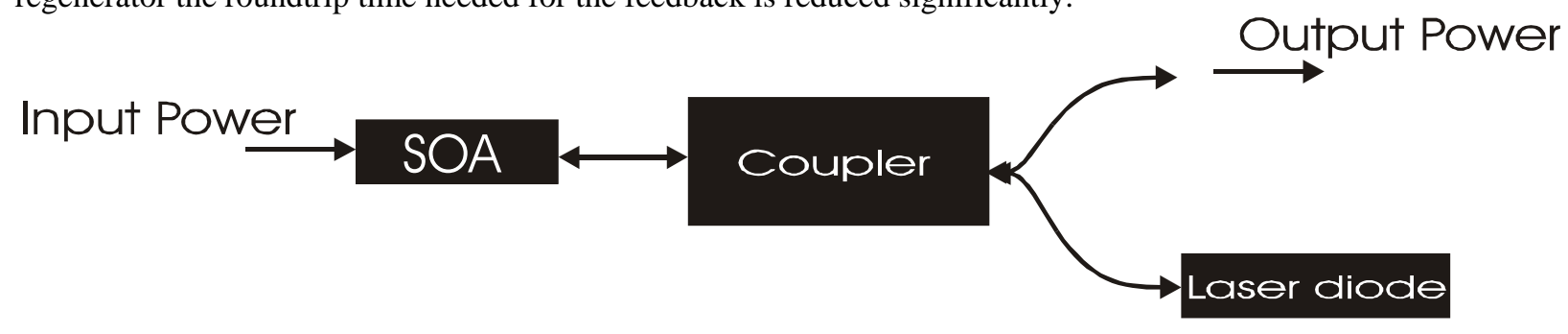

Fig. 1: Schematic of a semiconductor laser integrated with an SOA

In the experiments shown here we used a wavelength-selectable laser. In Fig. 2 a schematic representation of this device is given. It consists of an SOA that is connected to four laser diodes by means of a 1 by 4-coupler. Normally the light is coupled from lasers to SOA. In our case light is injected into the SOA and coupled out at the 
back facet of one of the lasers. One can already notice two distinct differences with the originally simulated and measured device. The coupling between laser diode and SOA is smaller which leads to less feedback and the output light has to pass through the laser before being measured.

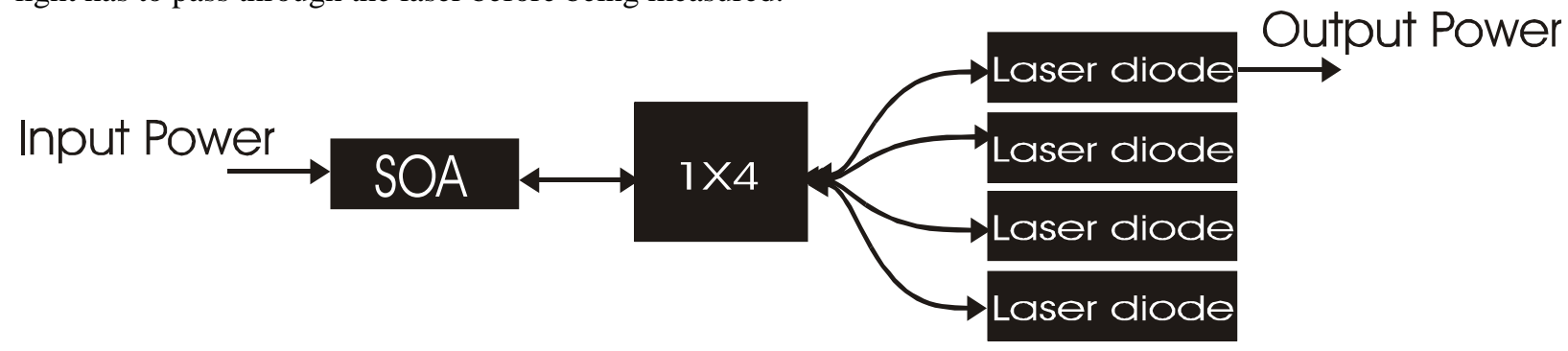

Fig. 2: Schematic of the wavelength-selectable laser used as regenerator

\section{Static measurements}

As a first step to experimentally characterise the new regenerator concept using the wavelength-selectable laser the static response has been studied. Light coming from a tunable laser source operating at a wavelength of $1553 \mathrm{~nm}$ is coupled into the component using a lensed fiber. At the back facet of the pumped laser diode operating at a wavelength of $1545 \mathrm{~nm}$ the light is coupled out again using a lensed fiber.

In Fig. 3 a decision characteristic is shown on which one can clearly see a step of about $5 \mathrm{~dB}$ in output power over an input power range of less than $1 \mathrm{~dB}$. The relatively low output power can be caused by the fact that the light is coupled out at the back facet of the component. This back facet has not been designed for optimal coupling but as a mirror for the laser diodes in its original purpose.

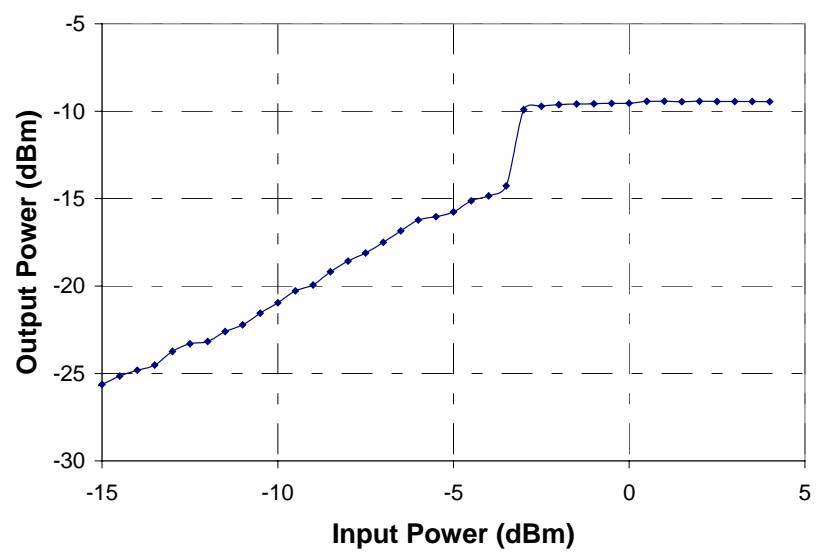

Fig. 3: Measured decision characteristic

\section{Dynamic measurements at $10 \mathrm{Gbit} / \mathrm{s}$}

In a next step dynamic measurements at $10 \mathrm{Gbit} / \mathrm{s}$ have been performed. The data signal is generated by modulating light from a tunable laser at a wavelength of $1555 \mathrm{~nm}$, with a PRBS of word length $2^{31}-1$. Before coupling to the component using a lensed fiber the light is sent through an EDFA, an attenuator and a 90/10 splitter. The EDFA and attenuator are used to control the power and quality of the light coupled into the component. The $10 \%$ fraction is used to monitor the input signal simultaneously with the output signal. At the back facet the light is again coupled out of the component using a lensed fiber. This signal is detected using a pre-amplified receiver.

In Fig. 4 input and output eye-diagrams at $10 \mathrm{Gbit} / \mathrm{s}$ are shown. It can be seen that an output extinction ratio of $10.3 \mathrm{~dB}$ was obtained for an input extinction ratio of $3.4 \mathrm{~dB}$. The input eye diagram was measured after the 90/10 splitter and with an extra attenuation of $6 \mathrm{~dB}$ resulting in a total attenuation of $16 \mathrm{~dB}$. The output eye diagram was measured with an attenuation of the input power of $14 \mathrm{~dB}$. Taking these attenuations into account one can see that the powers in the eye diagrams show good agreement with the difference between input and output power of the decision characteristic shown in Fig. 3. 


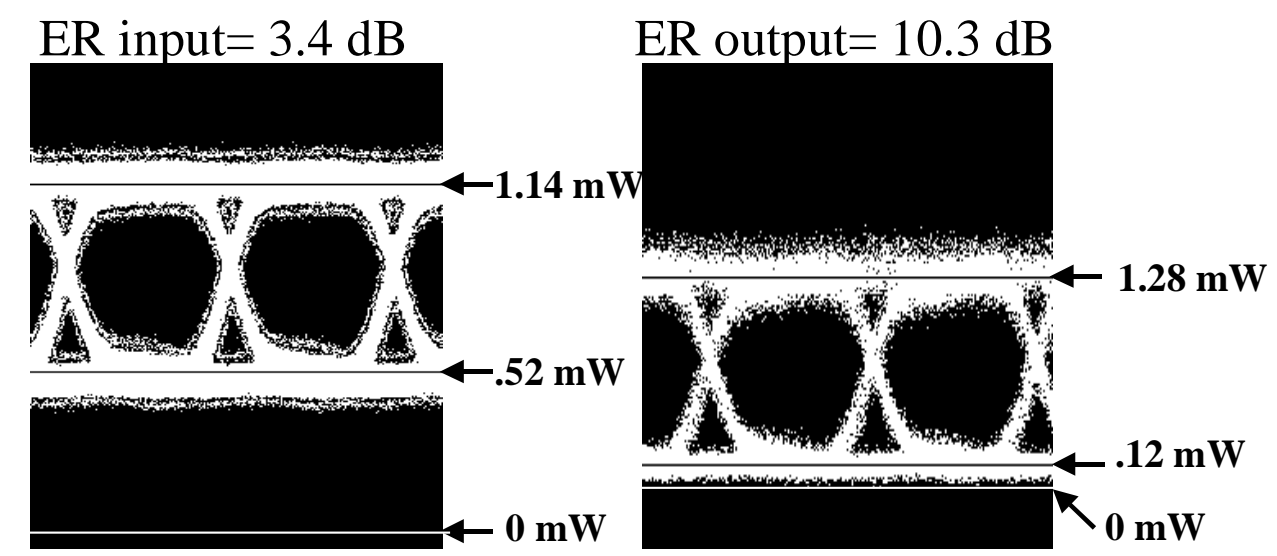

Fig. 4: Input and output eye diagrams

\section{Conclusion}

We have shown static and dynamic measurement results of an implementation with existing components of a regenerator based on the mutual optical feedback between a laser diode and an SOA. The static measurement shows a very steep transfer function. The dynamic measurements show extinction ratio improvement up to $6.9 \mathrm{~dB}$ at 10Gbit/s.

The circuit has a very simple structure and requires very little control. Moreover, since there is no interference involved the decision characteristic can be obtained over a wide wavelength range.

\section{References}

[1] K. Nonaka, Y. Noguchi, H. Tsuda, T. Kurokawa, "Digital signal regeneration with side-injection-light-controlled bistable laser diode as a wavelength converter”, IEEE Photonics Technology Letters, vol. 7, jan. 1995, pp. 29-31.

[2] M. Pantouvaki, M.J. Fice, R. Feced, E.P. Burr, R. Gwilliam, A.B. Krysa, J.S. Roberts, A.J. Seeds, “10-Gb/s all-optical 2R regeneration using an MQW Fabry-Perot saturable absorber and a nonlinear fiber”, IEEE Photonics Technology Letters, Vol. 16, Feb. 2004, pp. 617-619.

[3] D.A.O. Davies, A.D. Ellis, G. Sherlock, "Regenerative $20 \mathrm{Gbit} / \mathrm{s}$ wavelength conversion and demultiplexing using a semiconductor laser amplifier nonlinear loop mirror”, Electronics Letters, Vol. 31, June 1995, pp. 1000-1001.

[4] D. Wolfson, P.B. Hansen, A. Kloch, T.Fjelde, C. Janz, A. Coquelin, I. Guillemot, F. Garorit, F. Poignt, M. Renaud, “All-Optical 2R regeneration at $40 \mathrm{Gbit} / \mathrm{s}$ in an SOA-based Mach-Zehnder interferometer”, presented at the OFC-IOOC 1999, paper PD36 suppl

[5] J. De Merlier, G. Morthier, P. Van Daele, I. Moerman, R. Baets, “All-optical 2R-regeneration based on integrated asymmetric Mach-Zehnder interferometer incorporating MMI-SOA”, Electronics letter, vol. 38, Feb. 2002, pp. 238-239.

[6] O. Leclerc, B. Lavigne, E. Balmefrezol,P. Brindel, L. Pierre, D. Rouvillain, F. Seguineau, “ Optical regeneration at 40Gb/s and beyond”, J. Lightwave Technolog., vol. 21, Nov. 2003, pp. 2779-2790.

[7] W.D’Oosterlinck, G. Morthier, " Ultra-sharp optical decision characteristic from a laser diode integrated with a semiconductor optical amplifier”, in Optical Amplifiers and Their Applications / Integrated Photonics Research Topical Meetings on CD-ROM (The Optical Society of America, Washington, DC, 2004), JWB09

[8] W. D’Oosterlinck,G.Morthier,R. Baets, M.K. Smit, “ Very steep optical thresholding characteristic using a DFB laser diode and an SOA in an optical feedback scheme”, accepted for publication in Photonics Technology LettersAccepted

[9] K. Yashiki, K. Sato, T. Morimoto, S. Sudo, K. Naniwae, S. Ae, K. Shiba, N. Suzuki, T. Sasaki and K. Kudo, "Wavelength-Selectable Light Sources Fabricated Using Advanced Microarray-Selective Epitaxy," IEEE Photon. Technol. Lett., vol. 16, pp. 1619-1621, 2004.

\section{Acknowledgments}

This work has been supported by the European Commission through the IST-STOLAS project and by the Belgian DWTC through the IAP5/18 project. Wouter D'Oosterlinck and Sam Verspurten acknowledge the Flemish IWT for financial support. The authors would like to thank K. Kudo, K. Yashiki, K. Sato, and J. De Merlier from NEC Corporation for lending wavelength-selectable light sources. 